\title{
Avaliação das características da resina epóxi com diferentes aditivos desaerantes
}

\section{Evaluation of the characteristics of an epoxy resin with different degassing agents}

\author{
Alexsandro Oliveira ${ }^{1}$, Cristiane Miotto Becker ${ }^{1}$ e Sandro Campos Amico ${ }^{1}$ \\ ${ }^{1}$ Programa de Pós-graduação em Engenharia de Minas, Metalúrgica e de Materiais - PPGE3M, \\ Universidade Federal do Rio Grande do Sul - UFRGS, Porto Alegre, RS, Brasil \\ *cristiane.becker@senairs.org.br
}

\section{Resumo}

Este trabalho tem por objetivo a busca por técnicas alternativas para diminuir a incidência de vazios em resinas poliméricas e, por consequência, em materiais compósitos. Foram produzidas formulações diferentes utilizando resina epóxi à base de éter diglicidílico do Bisfenol A, endurecedor de poliamina alifática com e sem a adição de diferentes aditivos desaerantes a base de éteres e ésteres modificados. As formulações produzidas foram submetidas a condições extremas de agitação e processamento, sendo posteriormente avaliadas quanto a incidência de bolhas e vazios, densidade, propriedades mecânicas, morfológicas e térmicas. Os resultados foram bastante positivos quanto à diminuição da quantidade e do tamanho de poros pela utilização de técnicas de desgaseificação a vácuo e pelo uso de aditivos desaerantes.

Palavras-chave: resina epóxi, aditivos desaerantes, vazios.

\section{Abstract}

This study aims alternative techniques to reduce the incidence of voids in polymeric resins and in composite materials. Different formulations were prepared using epoxy resin based on bisphenol A diglycidyl ether and an aliphatic polyamine hardener, with and without the addition of different degasing agents based on modified esters and ethers. The formulations produced were subjected to extreme agitation and processing conditions and we evaluated the incidence of bubbles and voids, density, mechanical, morphological and thermal properties. The results were very positive. We observed the decrease in number and size of pores by the use of vacuum degassing techniques and degasing agent additives.

Keywords: epoxy resin, degassing agents, voids.

\section{Introdução}

A presença de vazios afeta severamente as propriedades das resinas poliméricas e, por consequência, as propriedades dos materiais compósitos produzidos com estas resinas, principalmente as propriedades mecânicas ${ }^{[1-4]}$. Em compósitos, os vazios podem se apresentar em vários formatos e tamanhos, sendo diversas as causas para a sua formação, entre elas, $o$ aprisionamento de gás (frequentemente gás úmido) durante o processo de impregnação dos reforços fibrosos com resina, os voláteis gerados durante a formulação da resina e bolhas de ar que podem ser aprisionadas durante a agitação de resinas de elevada viscosidade ${ }^{[5-9]}$.

A quantidade de bolhas de ar aprisionadas, por vezes, pode ser reduzida se o sistema de resina é sujeito a um processo de desgaseificação a vácuo ou pelo próprio uso do vácuo durante o processo de moldagem ${ }^{[10]}$. A busca por técnicas alternativas para reduzir a incidência destes defeitos leva ao desenvolvimento de aditivos químicos especiais, o que consiste na inclusão de um aditivo desaerante no sistema polimérico que deslocará as bolhas de ar para a superfície da resina, trabalhando em conjunto com o polímero em uma relação de compatibilidade e solubilidade e alterando sua tensão superficial. Neste sentido, trabalhos anteriores do grupo de pesquisa avaliaram o efeito do uso de um aditivo desaerante nas propriedades de compósitos de resina epóxi utilizando mantas e tecidos de fibras de vidro como reforço. Os resultados mostraram que a utilização do aditivo desaerante minimiza a formação de vazios, contudo, o trabalho sugere que os efeitos positivos de sua utilização poderiam ser mais bem apreciados caso a avaliação das propriedades fosse feita diretamente na resina epóxi, sem a presença de fibras ${ }^{[1]}$.

Assim, o objetivo deste trabalho foi avaliar o efeito da utilização de aditivos desaerantes diretamente nas propriedades de uma resina epóxi. Para tal, foram avaliados três tipos diferentes de desaerantes e uma combinação sugerida pelo fabricante. As formulações produzidas foram avaliadas através de medidas de densidade, propriedades mecânicas, morfológicas e térmicas. 


\section{Experimental}

\subsection{Materiais}

Foram utilizados resina epóxi à base de éter diglicidílico do Bisfenol A(Araldite LY 1316 BR, Huntsman), endurecedor a base de poliamina alifática (HY 1208, Huntsman) e aditivos desaerantes do fabricante Byk Chemie:

a) Desaerante isento de silicone à base de nafta de baixo ponto de ebulição e acetato de 1-metil-2-metoxietilo, denominado de Des A;

b) Desaerante isento de silicone à base de nafta de baixo ponto de ebulição, nafta hidrodessulfurada, butilglicolato e 2-butoxietanol, denominado de Des B;

c) Desaerante isento de silicone à base de nafta de baixo ponto de ebulição, nafta hidrodessulfurada, butilglicolato e acetato de 1-metil-2-metoxietilo, denominado de Des C.

\subsection{Preparação das formulações para determinação do aditivo desaerante de melhor desempenho}

No início de cada rota, as amostras de resina epóxi foram submetidas a um processo de agitação mecânica vigorosa de $500 \mathrm{rpm}$ por 5 min em um agitador $713 \mathrm{D}$ da marca Fisatom. Esta agitação prévia foi realizada a fim de se criar condições extremas no que diz respeito ao aprisionamento de bolhas de ar.

As formulações foram nomeadas conforme o processo utilizado para a retirada das bolhas de ar. Assim tem-se:

- Neat: Após a agitação inicial, não recebeu nenhum tratamento para a extração do ar aprisionado;

- Neat Deg: Após a agitação inicial, foi submetida à desgaseificação a vácuo por $1 \mathrm{~h} \mathrm{a} 60{ }^{\circ} \mathrm{C}$;

- DesA: Após a agitação inicial, foi adicionado o aditivo $\operatorname{DesA}(0,5 \% \mathrm{~m} / \mathrm{m}$ de resina+endurecedor $)$;

- DesB: Após a agitação inicial, foi adicionado o aditivo $\operatorname{DesB}(0,5 \% \mathrm{~m} / \mathrm{m}$ de resina+endurecedor $)$

- DesC: Após a agitação inicial, foi adicionado o aditivo DesC $(0,5 \% \mathrm{~m} / \mathrm{m}$ de resina+endurecedor $)$;

- DesAC: Após a agitação inicial, foi adicionado o aditivo DesA e DesC (respectivamente $0,5 \%$ e $0,3 \% \mathrm{~m} / \mathrm{m}$ de resina+endurecedor).

Nas amostras com aditivo desaerante, este foi adicionado após a agitação inicial, e então estas amostras foram submetidas novamente à agitação mecânica durante $5 \mathrm{~min}$, porém a uma velocidade de $200 \mathrm{rpm}$, apenas com o intuito de homogeneizar o aditivo no volume de resina. Após um repouso de $1 \mathrm{~h}$ à temperatura ambiente, adicionou-se o endurecedor na razão estequiométrica mássica $(87: 13)$ e realizou-se uma nova homogeneização da amostra (a $200 \mathrm{rpm}$ por $5 \mathrm{~min}$ ) que então foi vazada cuidadosamente em moldes de silicone. As duas formulações de controle foram submetidas ao mesmo processo de produção, com exceção da etapa de adição do desaerante. Contudo, na amostra desgaseificada a vácuo, a desgaseificação foi realizada após o processo inicial de agitação mecânica vigorosa e antes da adição do endurecedor.

\subsection{Confecção dos corpos de prova}

Os corpos de prova para os diferentes ensaios foram obtidos por casting em moldes de silicone. Após, os corpos de prova (CPs) foram curados à temperatura ambiente por $8 \mathrm{~h}$ e desmoldados, permanecendo em repouso durante 7 dias à temperatura ambiente para cumprir com o período de pós-cura. Por último, os corpos de prova foram lixados a seco em uma politriz, marca Pantec, modelo Polipan-U.

\subsection{Caracterização}

As seguintes análises foram realizadas nos diferentes sistemas curados:

a) Densidade: A densidade foi medida com o auxílio de um picnômetro de $50 \mathrm{ml}$ conforme norma ASTM D792. Foram realizadas 5 medidas para cada formulação.

b) Ensaio de Tração: Os ensaios de tração foram realizados em uma máquina universal de ensaios mecânicos EMIC DL 2000, operando com uma célula de carga de $2000 \mathrm{kgf}$ e uma velocidade de $5 \mathrm{~mm} / \mathrm{min}$, segundo a norma ASTM D3039. Estes ensaios foram realizados utilizando-se 10 corpos de prova para cada formulação

c) Ensaio de Flexão: O ensaio de flexão foi realizado com base na norma ASTM D790. Para este ensaio, realizado no mesmo equipamento EMIC DL 2000, foram ensaiados 10 corpos de prova para cada formulação.

d) Ensaio de Impacto: Foram realizados ensaios de impacto Izod em amostras sem entalhe seguindo a norma ASTM D256. Utilizou-se um equipamento CEAST, modelo Impactor II, com martelo de 2,75 J, e 10 corpos de prova para cada formulação.

e) Dureza Barcol: Para este ensaio, utilizou-se um durômetro Barcol digital da Bareiss, adequado para testes em plásticos rígidos. Foram realizadas 10 leituras na superfície de cada lado dos corpos-de-prova, seguindo a norma ASTM D2583.

f) Microscopia Óptica: Foi utilizado um microscópio óptico Nikon Eclipse ME 600 com câmera digital Samsung SDC-415 para avaliar a superfície de fratura dos corpos-de-prova submetidos ao ensaio de tração. Para tratamento de imagens foi utilizado o software ImageJ, para determinar o tamanho médio dos vazios presentes na amostra.

g) Calorimetria Diferencial de Varredura (DSC): Estas análises foram realizadas de forma a acompanhar o processo de cura das formulações. Desta forma, foram utilizadas formulações recém preparadas, antes do estágio de cura. O equipamento utilizado para esta análise foi um calorímetro modelo Q20 da marca TA Instruments. As formulações foram submetidas a esta análise, em atmosfera de nitrogênio, com o objetivo de avaliar suas características térmicas, tais como tempo de gel, temperatura do pico exotérmico e de início da faixa de cura para cada formulação. As análises foram realizadas com taxa de aquecimento de $10^{\circ} \mathrm{C} /$ min. As amostras foram acondicionadas em cápsulas de alumínio. 


\section{Resultados e Discussão}

As medidas de densidade (Figura 1) das amostras não mostram variações estatisticamente significativas, uma vez que, considerando o desvio padrão dos resultados, todas as amostras mostraram valores de densidade semelhantes. Apesar disso, pode-se sugerir uma tendência para o aumento da densidade das formulações submetidas ao processo de extração de bolhas de ar em comparação à formulação Neat (sem tratamento). Esses resultados já eram esperados, uma vez que o tratamento tem como finalidade minimizar o teor de bolhas de ar contido na formulação. Ainda, a amostra que apresentou o menor desvio padrão de medida e uma densidade ligeiramente maior foi a formulação Des B.

Os ensaios de tração (Figura 2) permitiram avaliar a resistência máxima, o alongamento na ruptura e o módulo de Young das diversas composições. O gráfico de resistência à tração (2A) apresenta as médias de resistência à tração para as diferentes amostras e, a partir da análise destes resultados, é possível observar que o tratamento de degasagem com ultrassom provoca alterações bastante significativas na resistência à tração, isso também é observado nas amostras que utilizaram aditivo desaerante.

Dentre essas amostras, o aditivo que mostrou melhores resultados foi o Des $\mathrm{B}$, confirmando os resultados obtidos nas medidas de densidade. Esse desempenho pode ser justificado devido à composição química deste desaerante, que por ser um éter glicol, atua como um tensoativo moderado, modificando a tensão superficial e dissolvendo os gases contidos na mistura, bem como outras substâncias que possam estar presentes. Ainda, essa formulação apresentou o melhor resultado de alongamento na ruptura (2B), o que mostra que este desaerante pode estar contribuindo com a tenacificação do material. Em contraste a isso, os resultados de elongamento na ruptura das formulações Des A, Des C e pela combinação destes dois desaerantes, levam a inferir que, além de não atuar de forma tão eficaz como na remoção dos vazios, a adição destes aditivos pode estar contribuindo para o aumento do número de ligações cruzadas, uma vez que a amostra se torna mais rígida. Os módulos de Young das amostras não apresentaram diferenças significativas (2C), sendo uma evidência de que os vazios presentes nas formulações não influenciam diretamente no módulo.

Nos ensaios de impacto (Figura 3), verificou-se que todas as formulações fraturaram de forma frágil, como esperado para uma resina termorrígida não-reforçada. Porém, percebe-se um pequeno aumento na resistência da maioria das formulações tratadas quando comparadas à formulação sem tratamento, o que indica a redução no número de sítios de falhas prematuras devido à presença dos vazios. A formulação Des AC apresentou o pior resultado, ficando com valores inferiores aos da formulação Neat. Este resultado vem corroborar o resultado de alongamento na ruptura, onde foi sugerido que esta combinação de aditivos está contribuindo para o aumento do número de ligações cruzadas na amostra, tornando-a mais frágil.

A dureza Barcol pode ser usada como um indicador simples do grau de cura de resinas termorrígidas, podendo servir como um parâmetro de controle de qualidade. Através deste ensaio, é possível determinar o tempo mínimo necessário para realizar a desmoldagem de uma peça, por

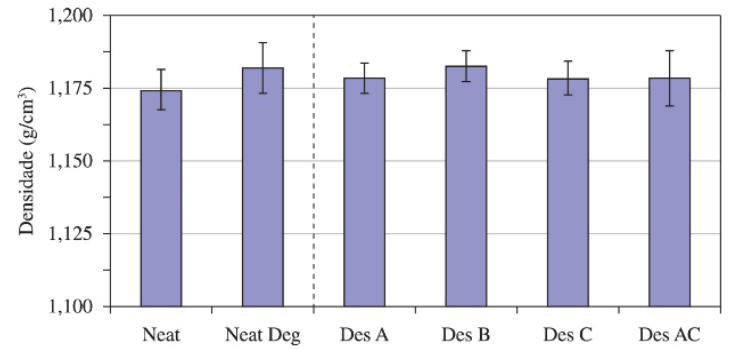

Figura 1. Valores de densidade das diferentes amostras.

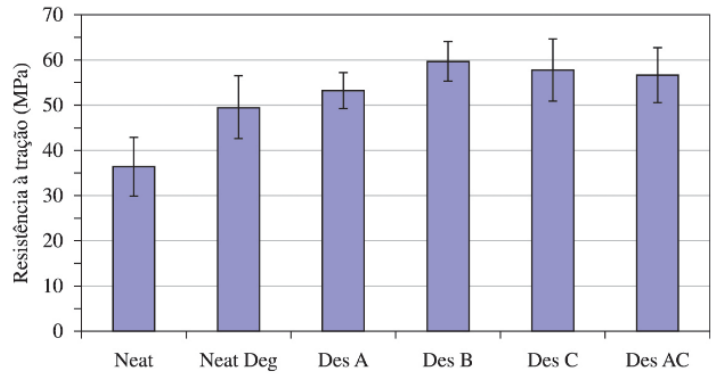

(a)

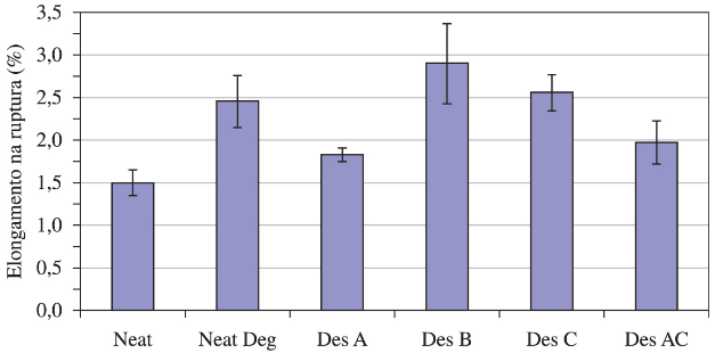

(b)

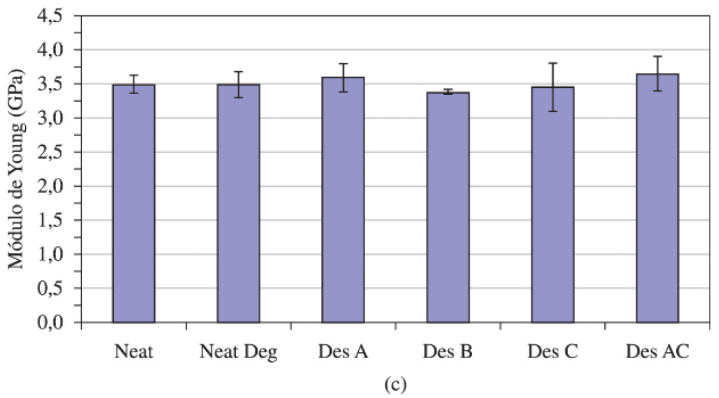

Figura 2. Valores médios de resistência à tração (a), elongamento na ruptura (b) e módulo de Young (c) para as diferentes amostras.

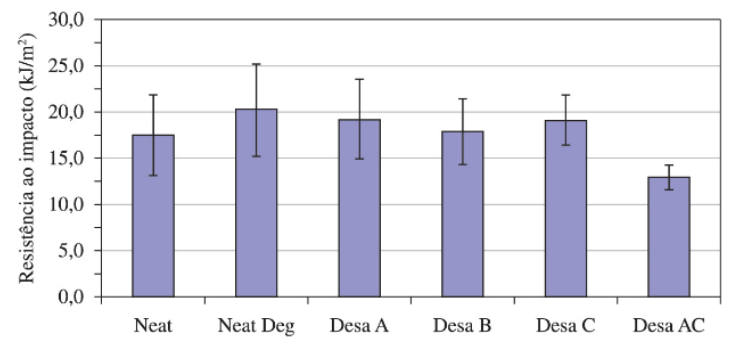

Figura 3. Valores médios de resistência ao impacto das formulações epóxi. 
exemplo. Conforme apresentado na Figura 4, as formulações apresentaram valores médios similares, o que indica que os aditivos não diminuem o grau de cura das resinas aditivadas.

Sabe-se que as resinas epóxi, depois de curadas, formam uma estrutura altamente reticulada e com isso espera-se uma fratura frágil quando a mesma é submetida a solicitações mecânicas. Assim, as Figuras 5-6 mostram micrografias da região de fratura das diferentes amostras. A análise destas microscopias revelou que os vazios encontrados na resina epóxi possuíam geometrias circulares ou elípticas, e que a fratura foi nucleada a partir destes vazios, ou de falhas préexistentes ${ }^{[12]}$. Por exemplo, na Figura 5a pode-se observar a região onde se iniciou a fratura (marcada com uma seta) e na Figura $5 b$ as bolhas de ar aprisionadas da formulação Neat.

$\mathrm{O}$ estudo do mecanismo de fratura revela que um corpo contendo uma falha terá sua tensão aumentada nas extremidades do eixo, por isso a redução do número de vazios bem como o tamanho dos mesmos é de grande interesse. Em função do alto grau de reticulação, a fratura de todas as formulações produzidas apresentou comportamento similar,

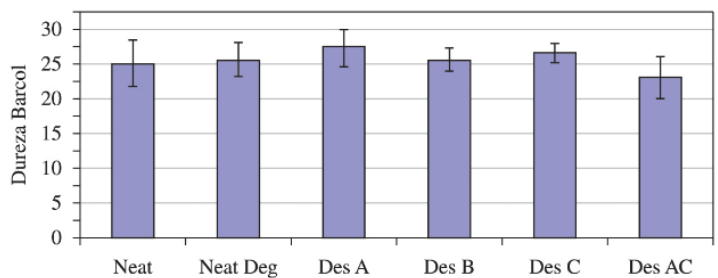

Figura 4. Representação dos valores médios obtidos para dureza Barcol.
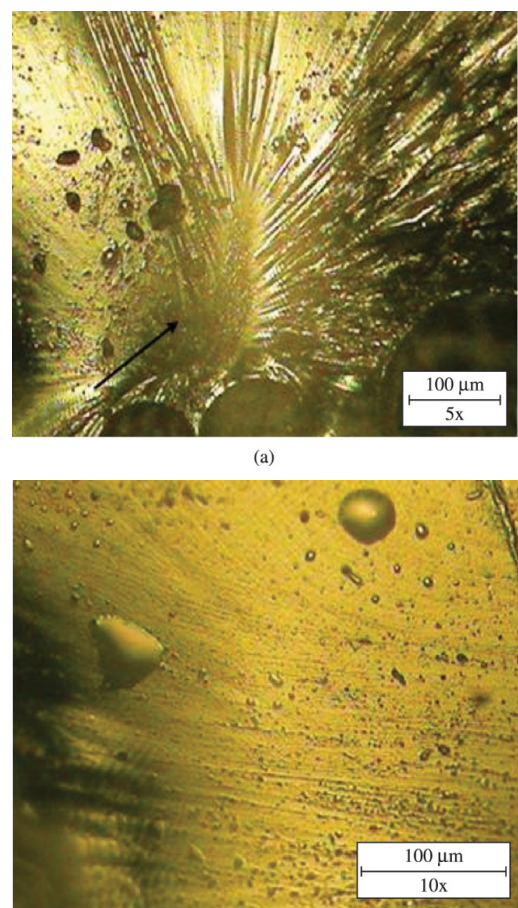

(b)

Figura 5. Micrografia óptica do (a) local da fratura frágil e (b) das bolhas de ar aprisionadas na resina (amostra Neat).

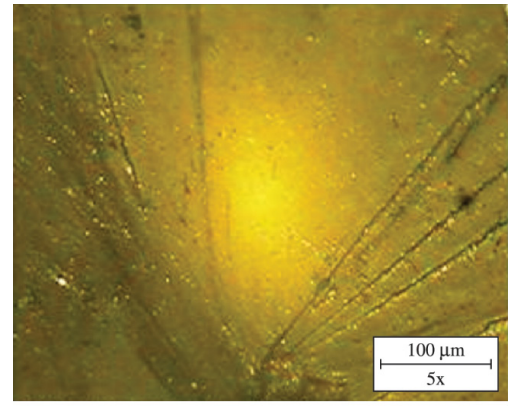

(a)

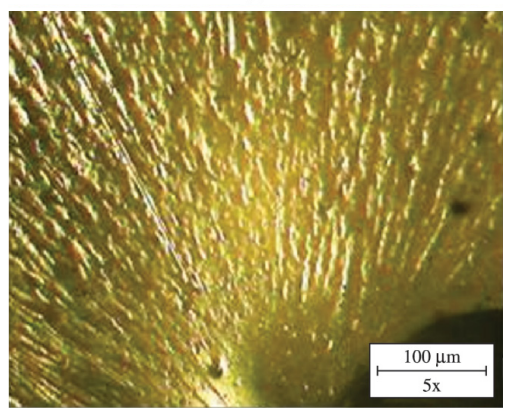

(b)
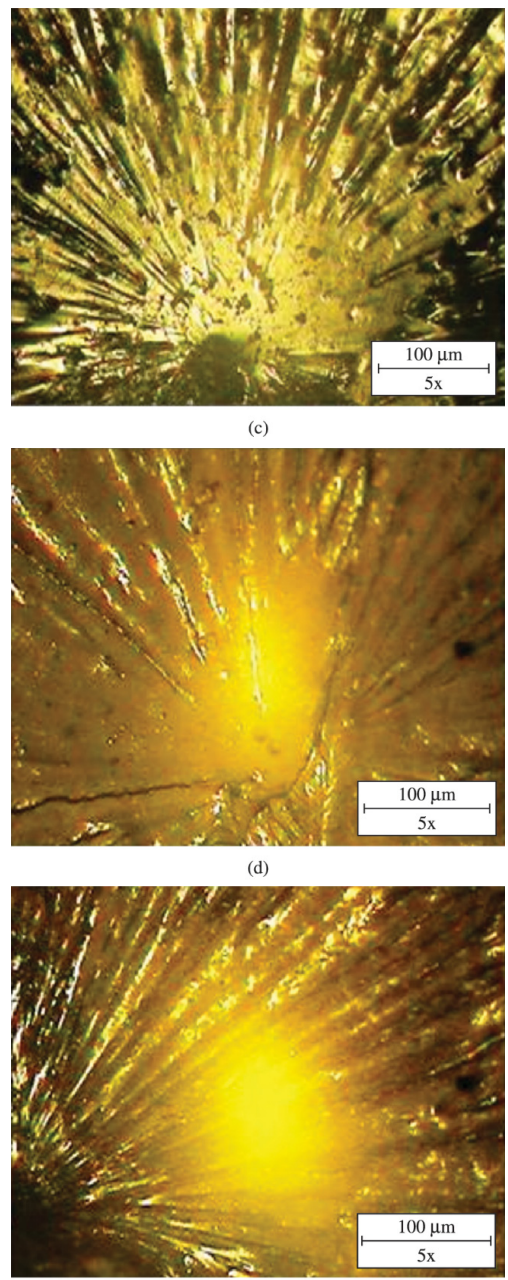

Figura 6. Microscopia óptica da fratura das formulações (a) Neat Deg, (b) Des A, (c) Des B, (d) Des C, (e) Des AC. 


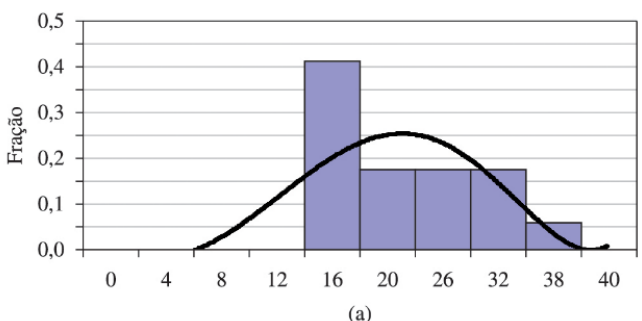

(a)

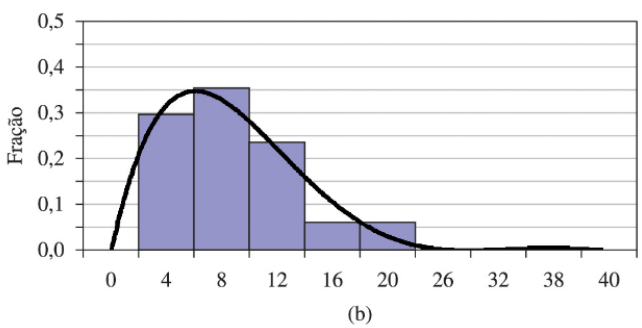

Figura 7. Tamanho médio de vazio $(\mu \mathrm{m})$ : (a) sistema Neat e (b) sistema DesB.

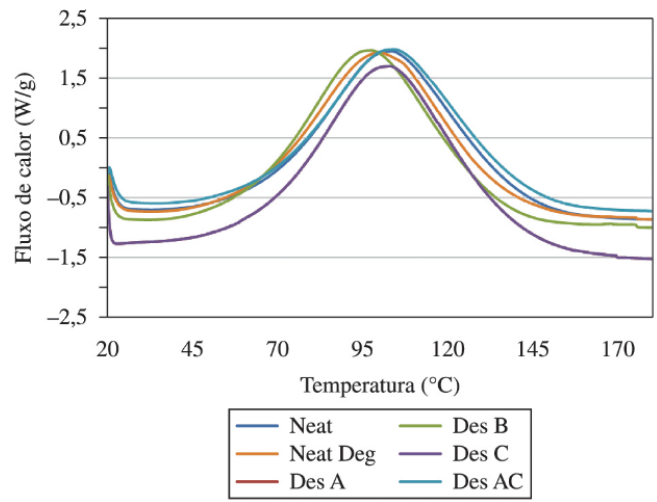

Figura 8. Gráfico de fluxo de calor $\times$ temperatura das diferentes formulações.

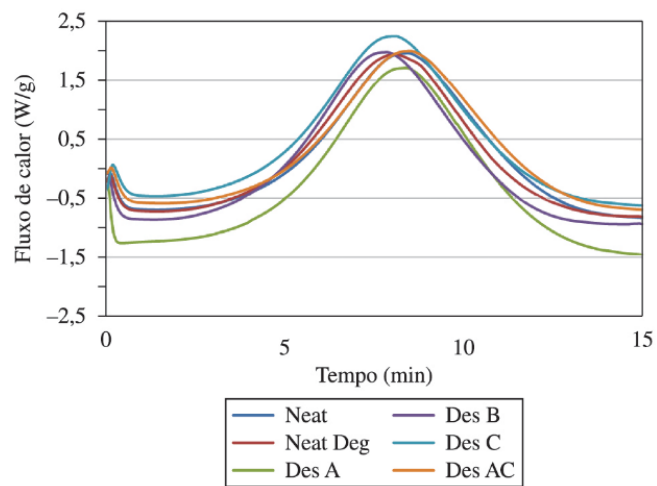

Figura 9. Gráfico de fluxo de calor $\times$ tempo das diferentes formulações.

Tabela 1. Dados dos ensaios de DSC das formulações analisadas.

\begin{tabular}{lccc}
\hline Amostra & $\boldsymbol{\Delta} \mathbf{H}(\mathbf{J} / \mathbf{g})$ & $\begin{array}{c}\text { Tempo de gel } \\
(\mathbf{m i n})\end{array}$ & $\mathbf{T}_{\text {pico }}\left({ }^{\circ} \mathbf{C}\right)$ \\
\hline Neat & 792,8 & 4,45 & 103,4 \\
Des A & 787,6 & 4,36 & 102,8 \\
Des B & 795,8 & 4,16 & 97,5 \\
Des C & 795,0 & 4,29 & 99,9 \\
Des AC & 769,2 & 4,36 & 104,2 \\
\hline
\end{tabular}

podendo-se verificar as chamadas "marcas de sargento" (Figuras 6a e b) que indicam a região de início da fratura.

Através de uma análise visual do tamanho dos vazios é possível perceber que o seu tamanho médio foi bastante reduzido para as formulações tratadas. Na formulação Neat (Figura 5), é possível verificar a existência de um grande número de vazios (bolhas) no interior do corpo de prova, o que não ocorre para a formulação, tanto a Net Deg quanto as contendo aditivos. Isto comprova a influência positiva da degasagem mecânica e do uso de aditivo desaerante na eliminação dos vazios na resina, e também justifica o melhor desempenho mecânico, principalmente em tração, destas amostras. Para confirmar esta observação, avaliou-se a distribuição de tamanhos de vazios nas amostras que apresentaram os piores (Neat) e os melhores resultados (Des B) nos ensaios mecânicos. Na Figura 7a, b, vê-se a distribuição de tamanho dos vazios e confirma-se que o diâmetro equivalente médio dos vazios foi bastante reduzido com o tratamento.

A análise por calorimetria diferencial de varredura (DSC) foi realizada para avaliar as condições de cura das diferentes formulações em condições de análise não-isotérmicas, com taxa de aquecimento de $10{ }^{\circ} \mathrm{C} / \mathrm{min}$. A Figura 8 mostra o acompanhamento da reação de cura com a elevação da temperatura e a Figura 9 mostra as mesmas reações de cura com o tempo de reação. Ambos os gráficos mostram um perfil de cura similar entre as formulações, com pequenas variações no tempo de gel, na entalpia de cura e na temperatura do pico exotérmico (Tabela 1). Pela análise dos valores de $\Delta \mathrm{H}$ residual é possível confirmar a informação sugerida nos resultado de alongamento na ruptura e resistência ao impacto, nos quais se sugere que a combinação dos desaerantes A e C está favorecendo o aumento de ligações cruzadas, uma vez que quanto menor o $\Delta \mathrm{H}$ residual, maior é a quantidade de ligações cruzadas.

\section{Conclusões}

A análise da influência da inclusão de aditivos desaerantes nas características do sistema epóxi frente ao tradicional processo de desgaseificação a vácuo demonstrou que a densidade da formulação Neat Deg foi superior à formulação sem qualquer tratamento para a redução das bolhas de ar. O mesmo ocorreu para a resistência à tração e o alongamento na ruptura das formulações que passaram pelo processo de extração de bolhas de ar, seja por desgaseificação ou pela adição de aditivos desaerantes.

Observou-se que, em geral, os melhores resultados foram alcançados pelas formulações Des B e Des C. No ensaio de impacto, observou-se melhora nos valores médios para os sistemas desgaseificados, resultado esperado em função da redução do tamanho médio de vazios. Na dureza Barcol não se observou alteração significativa o que mostra que a inclusão de aditivos não influenciou negativamente no grau de cura das formulações.

Assim, pode-se concluir que, dentre as formulações de desaerantes estudadas, a formulação Des B foi a que apresentou um melhor balanço das propriedades estudadas, sendo recomendado o seu uso como auxiliar no processo de remoção de bolhas para o sistema epóxi/endurecedor avaliado. 


\section{Agradecimentos}

Ao CNPq, CAPES e FAPERGS pelo apoio financeiro e a Universidade de Caxias do Sul pela realização dos ensaios de tração.

\section{Referências}

1. Schmidt, F., Rheinfurth, M., Horst, P., \& Busse, G. (2012). Multiaxial fatigue behaviour of GFRP with evenly distributed or accumulated voids monitored by various NDT methodologies. International Journal of Fatigue, 43, 207-216. http://dx.doi. org/10.1016/j.ijfatigue.2012.03.013.

2. Guo, Z. S., Liu, L., Zhang, B. M., \& Du, S. (2009). Critical void content for thermoset composite laminates. Journal of Composite Materials, 43(17), 1775-1790. http://dx.doi. org/10.1177/0021998306065289.

3. Grunenfelder, L. K., \& Nutt, S. R. (2010). Void formation in composite prepregs - Effect of dissolved moisture. Composites Science and Technology, 70(16), 2304-2309. http://dx.doi. org/10.1016/j.compscitech.2010.09.009.

4. Chambers, A. R., Earl, J. S., Squires, C. A., \& Suhot, M. A. (2006). The effect of voids on the flexural fatigue performance of unidirectional carbon fiber composites developed for wind turbine applications. International Journal of Fatigue, 28(10), 1389-1398. http://dx.doi.org/10.1016/j.jifatigue.2006.02.033.

5. Thomason, J. L. (1995). The interface region in glass fibrereinforced epoxy resin composites: 1 . Sample preparation, void content and interfacial strength. Composites, 26(7), 467-475. http://dx.doi.org/10.1016/0010-4361(95)96804-F.

6. Wiedemann, G., \& Rothe, H. (1986). Processing and fabrication. London: Elsevier Applied Science.
7. Costa, M. L., Almeida, S. F. M., \& Rezende, M. C. (2001). The influence of porosity on the interlaminar shear strength of carbon/epoxy and carbon/bismaleimide fabric laminates. Composites Science and Technology, 61(14), 2101-2108. http://dx.doi.org/10.1016/S0266-3538(01)00157-9.

8. Almeida, S. F. M., \& Nogueira Neto, Z. S. (1994). Effect of void content on the strength of composite laminates. Composite Structures, 28(2), 139-148. http://dx.doi.org/10.1016/02638223(94)90044-2.

9. Costa, M. L., Almeida, S. F. M., \& Rezende, M. C. (2001). Resistência ao cisalhamento interlaminar de compósitos com resina epóxi e diferentes arranjos das fibras na presença de vazios. Polímeros: Ciência e Tecnologia, 11(4), 182-189. http:// dx.doi.org/10.1590/S0104-14282001000400008.

10. Pizzutto, C. E., Suave, J., Bertholdi, J., Pezzin, S. H., Coelho, L. A. F., \& Amico, S. C. (2010). Mechanical and Dilatometric Properties of Carboxylated SWCNT/Epoxy Composites: Effects of the Dispersion in the Resin and in the Hardener. Journal of Reinforced Plastics and Composites, 29(4), 524-530. http:// dx.doi.org/10.1177/0731684408099924.

11. Oliveira, A., Becker, C. M., \& Amico, S. C. (2014). Efeito de aditivos desaerantes nas características de compósitos de epóxi/fibras de vidro. Polímeros: Ciência e Tecnologia, 24(1), 117-122. http://dx.doi.org/10.4322/polimeros.2014.059.

12. Hobbiebrunken, T., Fiedler, B., Hojo, M., \& Tanaka, M. (2007). Experimental determination of the true epoxy resin strength using micro-scaled specimens. Composites. Part A, Applied Science and Manufacturing, 38(3), 814-818. http://dx.doi. org/10.1016/j.compositesa.2006.08.006.

Enviado: Mar. 11, 2014 Revisado: Ago. 18, 2014 Aceito: Out. 07, 2014 